\title{
A Newborn with a Blasckoid-like Distribution Rash: Never Forget to Use the Dermoscope
}

\author{
Federica Filippi ${ }^{1}$, Marco Adriano Chessa ${ }^{1}$, Federico Bardazzi ${ }^{1}$, Iria Neri ${ }^{1}$ \\ 1 Dermatology Unit, IRCSS Policlinico di S. Orsola, Department of Experimental, Diagnostic and Specialty Medicine, University of Bologna, \\ Bologna, Italy
}

Citation: Filippi F, Chessa MA, Bardazzi F, Neri I. A newborn with a blasckoid-like distribution rash: never forget to use the dermoscope. Dermatol Pract Concept. 2022;12(1):e2022008. DOI: https://doi.org/10.5826/dpc.1201a08

Accepted: April 25, 2021; Published: January 2022

Copyright: $\bigcirc 2022$ Filippi et al. This is an open-access article distributed under the terms of the Creative Commons Attribution-NonCommercial License (BY-NC-4.0), https://creativecommons.org/licenses/by-nc/4.0/, which permits unrestricted noncommercial use, distribution, and reproduction in any medium, provided the original authors and source are credited.

Funding: None.

Competing interests: None.

Authorship: All authors have contributed significantly to this publication.

Corresponding author: Marco Adriano Chessa, MD, Dermatology Unit, Department of Experimental, Diagnostic and Specialty Medicine, University of Bologna, Bologna, Italy. E-mail: marco.adriano.chessa@gmail.com

\section{Case Presentation}

A 30-day-old newborn presented with a 7-day history of an erythematous-crusted rash with a blasckoid-like distribution on his arm and lower limb (Figure 1, A and B). The baby was sleeping well, and systemic symptoms were absent. A complete blood exam showed no abnormalities except for a mild eosinophilia. Dermoscopy revealed crusted scabies (Figure 1C). The patient was treated with permethrin 5\% cream, applied and left on the skin for 4 hours for 2 consecutive nights and then again for 2 consecutive nights after 7 days. Other family members were given the same treatment regimen, permethrin $5 \%$ cream, applied and left on the skin for 8 hours.

\section{Teaching Point}

Norwegian or crusted scabies is a variant of scabies with a massive infestation of Sarcoptes scabiei. Most cases are reported in immunocompromised patients; however, in an immature immune system, overcrowded living conditions can lead to the appearance of the disease in newborns. Our case is peculiar because of the absence of symptoms and the papulo-erythematous blasckoid-like presentation [1]. In these cases, dermoscopy has a central role in quickly arriving at a diagnosis and avoiding unnecessary investigation [2]. 


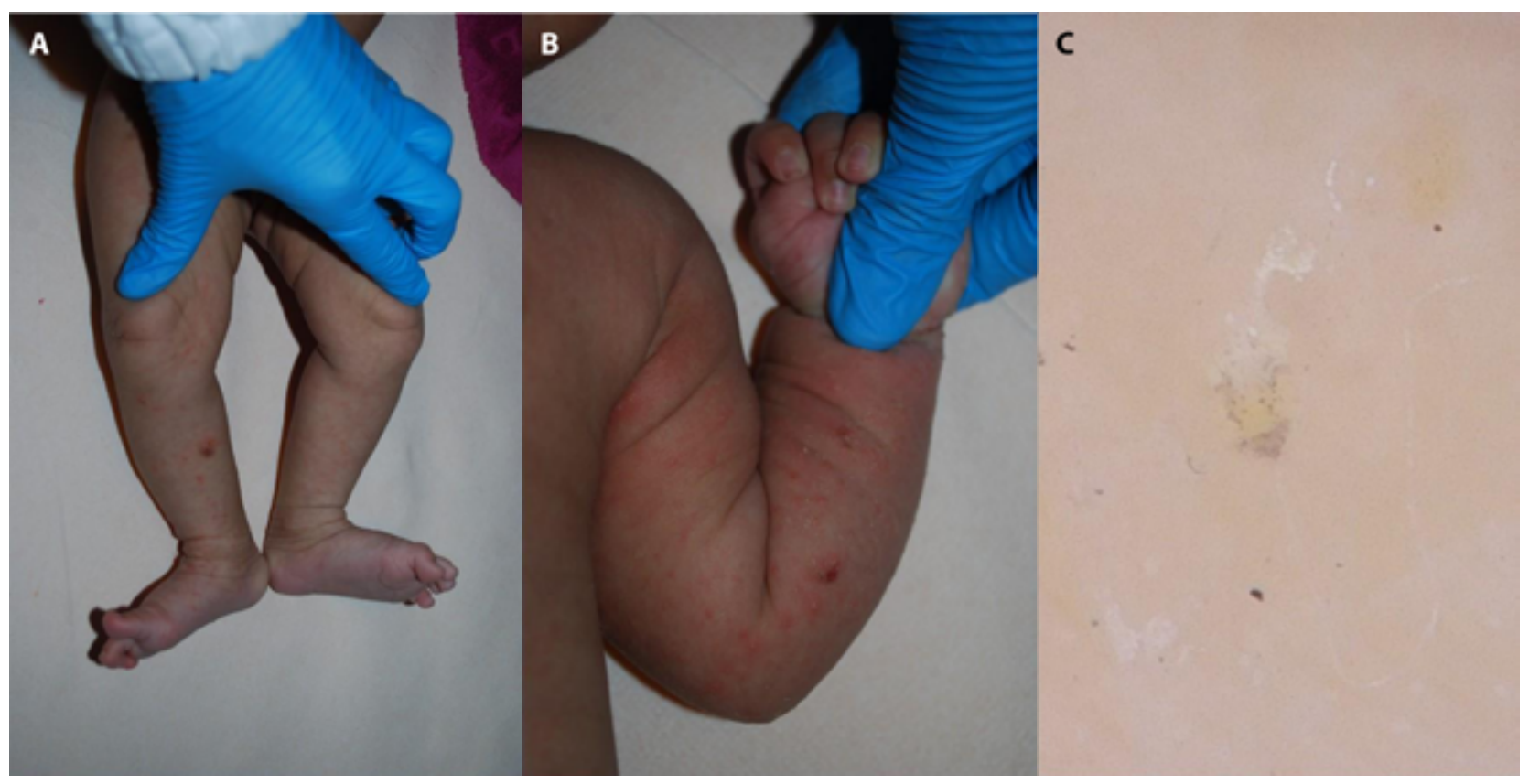

Figure 1. Detail of the erythematous-crusted rash.and. (A) Right lower limb. (B) Left arm with a blasckoid-like distribution. (C) The dermoscopic examination at a magnification of $\times 10$ revealed the clue for diagnosis: typical small brown pigmented triangular structures, the so-called hang-glider sign, in addition to S-shaped white burrows or jet-liner sign were detected, leading to the diagnosis of crusted scabies.

\section{References}

1. Neri I, Chessa MA, Virdi A, Patrizi A. Nodular scabies in infants: dermoscopic examination may avoid a diagnostic pitfall. J Eur Acad Dermatol Venereol. 2017;31(12):e530-e531. DOI: 10.1111/jdv.14401. PMID: 28609546.
2. Engelman D, Yoshizumi J, Hay RJ, et al. The 2020 International Alliance for the Control of Scabies Consensus Criteria for the Diagnosis of Scabies. Br J Dermatol. 2020;183(5):808820. DOI: 10.1111/bjd.18943. PMID: 32034956. PMCID: PMC7687112. 